\title{
The Effect of Visual Functions on Target Discrimination and Shooting Skill in Military Students
}

\author{
Saeedi Anari Ghassem¹, Makateb Ali ${ }^{2 *}$ \\ ${ }^{1}$ Department of Ophthalmology, Ardabil University of Medical Sciences, Ardabil, Iran \\ ${ }^{2}$ Department of Ophthalmology, AJA University of Medical Sciences, Tehran, Iran
}

*Corresponding author: Ali Makateb, Eye Research Center, Ophthalmology

Department, AJA University of Medical Sciences, Tehran, Iran.

Received Date: July 25, 2020

Published Date: August 27, 2020

\begin{abstract}
Introduction: The ability to fight with enemy with a gun (shooting) is one of the basic skills of military forces. The aim of this study is to demonstrate the effect of visual skills such as visual acuity, stereo acuity, color vision, contrast sensitivity and eye- hand dominance distribution on targeting and shooting scores in military forces.
\end{abstract}

Methods and materials: This is a cross sectional study. The sample group was random students of a military college in Tehran, Iran. After filling out consent forms the students were referred to the military hospital for examination. The mean age was between 18-28 years old. Cases with prior history of eye disease and surgery or neurological problem were excluded. The shooting score of each person and their best corrected visual acuity (BCVA) were recorded. Test results of stereo acuity, color vision and contrast sensitivity of each case at the table was recorded and compared with shooting scores. The data was analyzed with SPSS (version 19) software.

Results: A total of 100 cases were enrolled in this study. There was a direct relationship between visual acuity and target shooting skills in the military students. It was found that people who had better visual acuity, had also better shooting scores.

Conclusion: The results showed that people who have better visual acuity and stereo acuity have better shooting scores.

Keywords: Shooting, Visual acuity, Targeting

\section{Introduction}

Athletes need visuals skills in addition to other skills like mental skills, sports intelligence, and cognitive skills. There are little studies about "vision skills" among all sports kills in our country [1]. Rifle shooting like other sport fields requires physical and mental fitness. Properuse of visual skills, the correct judgment about speed and distance of the observed object, affects the proper functioning of the shooting. If a person with appropriate physical fitness could not use all its visual information, he/she cannot use the needed potential for shooting effectively. High Strength, speed and agility cannot off set in complete visual information. Studies showed that visual skills level are higher in successful athletes than amateur athletes [2]. Visuals kills include: Visual Acuity-Contrast Sen
sitivity-Color Discrimination-Stereoacuity-Fusion Flexibility (Binocularity)-Focus Flexibility (Accommodation)- Central/ Peripheral awareness-Visual Response Time-Visual Concentration-Visual Adjustability [1].

\section{Visual acuity}

The ability to see fixed targets at a distance which is measured as single and binocular by Smellen chart [3]. Contrast sensitivity: the system's ability to process visual and spatial information about the object and its background in different lighting conditions and usually used for binocular vision with functional made in athletes [4]. This test is done by CSVE 1000 chart. 


\section{Color vision}

The ability of color range distinction in shooting ability. D15 Frans worth test is used to test color vision [5].

\section{Stereo-Acuity}

This test gives better data about coordination of both eyes. Since judgments about the spatial position are one of the important tasks of the shooter, evaluation of stereo-acuity seems logical in them. For this purpose, TNO Stereo test is used [6-8].

\section{Ocular Dominance and Handedness and its effect on the performance of Shooter}

Roughly $60 \%$ of the population has been shown to be right-handed, 30\% left-handed and 10\% ambidextrous [9-13] Similarly approximately $67 \%$ of the population have been shown to have right eye dominance [14].

Originally, researchers thought that ocular dominance was related to handedness or foot dominance, especially as in both handedness and ocular dominance, there seems to be a strong predisposition towards favoring the right side of the body. Researchers have now agreed that while handedness and foot dominance have been found to be highly correlated with each other, they are neither in deceptive nor predictive of ocular dominance. Despite a vast amount of time and effort that has been invested in understanding the physiological basis of ocular dominance and its functional roles, little agreement between researchers has been reached [15-18] Results showed a high performance of all visual skills in professional athletes (elite) and also recorded norms showed the importance of visual skills in the sports performance of an athlete [19].

In 2009, Hatch and colleagues examined the effect of visual acuity in targeting and shooting. The results of visual skills in targeting showed that some skills such as visual acuity, contrast sensitivity, stereo acuity, cooperation between the body and the eyes and the time of visual response are the most important skills in shooting [1].

In 2006, Buys and Ferreir did a study to prepare the norms with the aim of providing visual skills in professional athletes (elite) especially in shooting. The results showed that all elite athletes has a superior visual system [20]. The aim of each visual assessment is to provide the best conditions that cause best visual function for activities of daily living. The athletic fitness and healthy vision of the army forces are very important in their sport skills. The aim of this study was to show the effect of visual skills such as visual acuity, stereo acuity, color vision, contrast sensitivity and eye-hand dominant distribution on targeting and shooting skills in the army forces.

\section{Materials and Methods}

100 military students of a college in Tehran, Iran were selected randomly and enrolled in this study. After filling the consent form, they refer red to military hospital for examination. The mean age was between 18-28 years old. Cases with any history of eye disease and surgery or neurological problem were excluded. The scores of shooting of each case and their best corrected visual were recorded. Test results of each case at the table was recorded and compared with shooting scores. Participants were truly familiar with G3 rifle 20 bullets which were given to them for test. Total scores calculated from 100 for ease of statistical analyses. The results of each visual test were recorded in a special question naire. The visual test results were compared with the shooting scores. Measured Visual Skills were:

1-Static visual acuity: It was measured in monocular and binocular position by Smeller chart. Visual acuity range was 10/ 20 to $20 / 200$.

2-Stereo- acuity was measured by TNO Stereo test.

3-Contrast sensitivity (high myopic) was performed in 10. 6 lux with CSVE 1000 chart

4-Colorvision: D15FQXG3YzHBdnscohjWesugYoyTV45SQRwnas the arrange ment test.

5-Eye dominance

The data analyzed by SPSS software version 19 .

\section{Results}

A total of 100 participants were enrolled in this study. Recorded visual acuity of dominant eye in each group is shown in Table1. In the shooting stage, the scores of 32 participants were $80-100,36$ participant $60-80,16$ participant $40-60,8$ participant $20-40,5$ participant 10-20 and 3 participant was 0-10. Evaluating the eye-hand co-ordination showed 65 (65\%) participant had right eye-hand dominance and 19(19\%) participant had left.

The color vision test recognized 90 normal (90\%), 4 participants with Protanopia/ protanomaly (4\%), 5 participants with Deuteranopia/ deuteranomalia (5\%), and 1 participant with Tritanopia/ tritanomaly (1\%). In stereo-acuity test 65 participant $(65 \%)$ had= 240 score and 35(35\%) Participant had $>240$.

Chi-square test showed that visual acuity had a significant relationship with shooting and targeting skills. It was found that people who have better visual acuity have better shooting. (X2=131.36, $\mathrm{df}=5, \mathrm{p}<0 / 001$ ) Table2. There was verse relationship between stereo- acuity and shooting skills. It means lower score of stereo-acuity test, better shooting skills. $(\mathrm{p}<0 / 001, \mathrm{r}=-0 / 369)$. So, who had better stereo-acuity, had higher scores in shooting. The results showed that there is not a significant statistical relationship between shooting skills and color vision. $(X 2=9.17, d f=15, p=0 / 7)$. It could be due to black and white target used for shooting. Also, there was not statistical relationship between eye-hand co-ordination and shooting skills but the participant with right eye-hand coordination had better scores. $(\mathrm{p}=0.96, \mathrm{z}=-0.048)$. Table3. Contrast sensitivity test rec- 
ognized 43 participants (43\%) with 1.95, 34 and 23 (34\%and23\%) trast sensitivity and shooting skills was not statistically significant. with $>2$ and 1.8-1.9 correspondingly. The relationship between con$(\mathrm{p}=0 / 11, \mathrm{r}=0 / 29)$.

Table 1

\begin{tabular}{|c|c|c|}
\hline Visual Acuity(BCVA) & Frequency & Percent \\
\hline 10 -Oct & 57 & $57 \%$ \\
\hline 10 -Sep & 22 & $22 \%$ \\
\hline 10 -Aug & 8 & $8 \%$ \\
\hline 10 -Jul & 3 & $3 \%$ \\
\hline $10-J u n$ & 5 & $5 \%$ \\
\hline 10 -May & 5 & $5 \%$ \\
\hline
\end{tabular}

Table 2: Best corrected visual acuity relationship with shooting skills.

\begin{tabular}{|c|c|c|c|c|c|c|c|}
\hline \multirow{2}{*}{ visual Acuity } & \multicolumn{6}{|c|}{ Shooting Scores } & \multirow{2}{*}{ Total } \\
\hline & $0-10$ & 20-0ct & $20-40$ & $40-60$ & $60-80$ & $80-100$ & \\
\hline 5 & 3 & 2 & 0 & 0 & 0 & 0 & 5 \\
\hline 6 & 0 & 3 & 2 & 0 & 0 & 0 & 5 \\
\hline 7 & 0 & 0 & 3 & 0 & 0 & 0 & 3 \\
\hline 8 & 0 & 0 & 2 & 4 & 2 & 0 & 8 \\
\hline 9 & 0 & 0 & 0 & 2 & 14 & 6 & 22 \\
\hline 10 & 0 & 0 & 0 & 11 & 19 & 27 & 57 \\
\hline Total & 3 & 5 & 7 & 17 & 35 & 33 & 100 \\
\hline
\end{tabular}

Table 3: Eye- hand coordination and shooting scores.

\begin{tabular}{|c|c|c|c|c|c|c|c|}
\hline \multirow{2}{*}{$\begin{array}{c}\text { Shooting Scores } \\
\text { Eye-hand Dominance }\end{array}$} & \multicolumn{6}{|c|}{ Shooting Scores } & \multirow{2}{*}{$\begin{array}{c}\text { Total } \\
65\end{array}$} \\
\hline & 10 & 20 & 40 & 60 & 80 & 100 & \\
\hline Right Eye and hand dominancy & 3 & 3 & 6 & 12 & 18 & 23 & 19 \\
\hline Left Eye and hand dominancy & 0 & 2 & 1 & 4 & 8 & 4 & 7 \\
\hline Right eye with the left hand dominancy & 0 & 0 & 0 & 1 & 3 & 3 & 9 \\
\hline Left eye with the right hand dominancy & 0 & 0 & 0 & 0 & 6 & 3 & 100 \\
\hline Total & 3 & 5 & 7 & 17 & 35 & 33 & 65 \\
\hline
\end{tabular}

\section{Discussion}

This cross-sectional study assessed the effect of visual skills such as visual acuity, stereo-acuity, color vision, contrast sensitivity and eye-hand coordination in targeting and shooting skills in military forces. According to the results of this study the people who have better visual acuity would have better shooting scores. In Hatch study in 2009, the effect of visual acuity on targeting and shooting was studied. The study showed that people who have the visual acuity better than 0.2 LOGMAR are clearly more successful in targeting and shooting than whom with 0.7LOGMAR or worse. According to this study, America's Army considered minimum standard of 20/40 or better (BCVA) to identify the target [21].

Daniel J Garland explained that visual acuity refers to the ability to make Fine visual discriminations among objects in the visual field. Although it seems obvious that good visual acuity would enhances port performance buthow ever, Bauscher, Garner, and Martin reported a large percentage of athletes maintain high performance with in adequate visual acuity. Additional researchers have come to the same conclusion, suggesting visual acuity is not related to athletic ability [22].

In our results, $72 \%$ of participants had right eye dominance. Also $32 \%$ of participants had eye-hand Coordination. Our results showed that the participants with right eye-hand dominance were more successful. Many studies surveyed the relationship between dominant eye and performance in various fields such as sports and education. Many of these studies found dominant eye as an important factor in determining the function al level but others showed no effect [23-26]. So, more studies are needed to show the effect of dominant eye in various functions.

The results of our study didn't show a significant relationship between color vision and shooting skills. The cause may be due to black and white target used for shooting. So, we can't consider a definite Statistical relationship between color vision and shooting skills.

The results of our study showed that stereo-acuity is better in participants who gained higher scores in shooting and vice versa. Several studies have suggested that better stereo acuity causes bet- 
ter activities such as threading needle, catch the ball and bottling with in the pan. Some individuals have a particular need to understand three-dimensional view, such as jobs using binocular microscopes are used. Athletes are other groups because it is needed to make judgments about the spatial position in many of the sports [27].

There was not significant statistical relationship between contrast sensitivity and shooting skills. According to the definition Contrast sensitivity is the ability to process visual and spatial information about the object and its background in different lighting conditions. But contrast sensitivity is used to see objects details in low-contrast situation like coming down the stairs, direction and movement in low light (in dust, rain and fog, snow fall and evening) Because our study was done in sunlight, so this result can't be definite. We suggest more studies to evaluate the effect of contrast sensitivity in shooting skills after omitting possible confounders' factors.

\section{Acknowledgement}

\section{None}

\section{Conflict of Interest}

No Conflict of Interest.

\section{References}

1. StrydomB, FerreiraJT (2010) The role of vision and visual skills in archery. Safr Optom 69(1): 21-28.

2. Jones LF III,Classe JG, Hester M (1996) Association between eye domin ance and training for rifle marksmanship: A Pilot Study. J Am Optom Assoc 67(2): 73-76.

3. Buys JHC, Ferrira JF (2010) the Development of Norms and Protocols in Sports Vision Evaluations. Safer Optom 69(1): 21-28.

4. Gregg JR (1987) Vision and sport an introduction Stoneham Butter worth P: $17-32$

5. Elliott DB (1993) Evaluating visual function in cataract. Optom Vis Science 70(11): 896-902.

6. Hazel CA (1995) The efficacy of sport vision practice and its role in clinical optometry. Clin Exp Optom 78: 98.

7. Starkes JL, Deakin J (1984) Preception in sport: a cognitive approach to skill ed performance in Straub WF, Williams JM, editor:cognitive sport psychology. Lancing NY sport Science Association. Pp 115-128.

8. Abernethy B (1986) Enhancing sport performance through clinical and experimental optometry. Clin Exp Optom 69: 189.

9. Eyre MB, Schmeckle MM (1933) A studies of handedness, eyedness, and footedness. Child Develop 4: 73-78.
10. Jasper HH, Raney ET (1937) The phitest of lateral dominance. Am J Psychol 49: 450-457.

11. Miles WR (1929) Ocular doming a demonstrated by unconscious sighting. J Exper Psychol 12: 113-126.

12. Berner GE, Berner DE (1953) Relation of ocular dominance, handedness, and the controlling eye in binocular vision. A MA Arch Ophthalmol 50(5): 603-608

13. Noll JE (1990) Vision considerations in shooting sports. N Eng J Optom 42: 675-681.

14. Miles WR (1930) Ocular dominance in human adults. J Gen Psychos 3: 412-420.

15. Pointer JS (2001) Sighting dominance, handedness, and visual acuity preference: three mutually exclusive modalities? Ophthalmic Physiol Opt 21(2): 117-126.

16. Laby DM, Kirschen DG (2011) Thoughts on ocular dominance- is it actually a preference? Eye Contact Lens 37(3): 140-144.

17. Classe JG, Daum K, Semes L, Wisniewski J, Rutstein R ,et al. (1996) Association between eye and hand dominance and hitting, fielding and pitching skill among players of the Southern Baseball League. J Am Optom Assoc 67(2):81-86.

18. Aswathappa I ,Kutty K, Annamalai N (2011) Relationship between handedness and ocular dominance in healthy young adults-astudy. Int ] Pharm Biomed Res 2: 76-78.

19. Laborde S, Dosseville FEM, Leconte P, Margas N (2009) Interaction of hand preference with eye dominance on accuracy in archery. Percept mot skills 108(2): 558-564.

20. J H C Buys and J T Ferreira (2008) The development of protocols and norms for sports vision evaluations. S Afr Optom 67(3): 106-117.

21. Brian C, Hatch, David J, Hilber (2009) The Effects of Visual Acuity on Targe Discrimination and Shooting Performance. Optom Vis Sci 86(12): E1359-E1367.

22. Daniel J, Garland (1990) sport expertise: the cognitive advantage. Perceptual and Motor Skill 70(3 pt 2): 1299-1314.

23. Classe J G, Daum K, Semes L, J Wisniewski, R Rutstein, et al. (1996) Association between eye and hand dominance and hitting, fielding and pitching skill among players of the Southern Base ball League. J Am Optom Assoc 67(2): 81-86.

24. Newman SP, Wadsworth JF, Archer R (1985) Oculardominance, reading, and spelling ability in school children. Br J Ophthalmol 69(3): 228-232.

25. Suleman R, Yang T, Paige J, Sheila Chauvin, Jaime Alleyn, et al. (2010) Hand-eye dominance and depth perception effects in performance on a basic laparoscopic skill set. JSLS 14(1): 35-40.

26. Laby D M, Kirschen D G (1999) The effect of binocular disparity on the determination of ocular dominance. Presented to the annual meeting of the American Association for Pediatric Ophthalmology and Strabismus, Toronto, Canada.

27. Grif in Binocular Vision Anomalies Professional press USA 1988. 